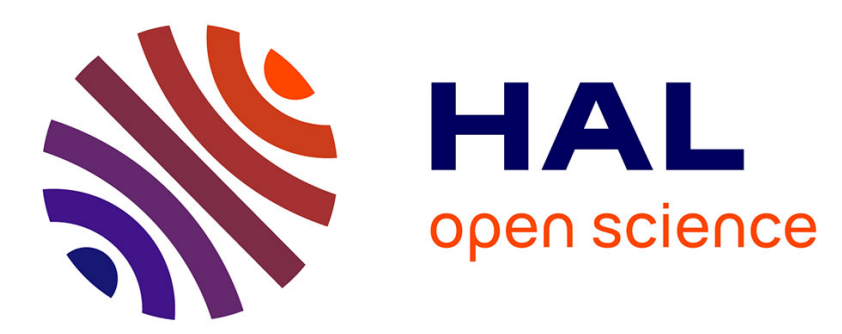

\title{
Ion Velocity Distribution Function Investigated Inside an Unstable Magnetized Plasma Exhibiting a Rotating Nonlinear Structure
} C. Rebont, N. Claire, Th. Pierre, F. Doveil

\section{- To cite this version:}

C. Rebont, N. Claire, Th. Pierre, F. Doveil. Ion Velocity Distribution Function Investigated Inside an Unstable Magnetized Plasma Exhibiting a Rotating Nonlinear Structure. Physical Review Letters, 2011, 106 (22), pp.9007 - 9018. 10.1103/physrevlett.106.225006 . hal-01787133

\section{HAL Id: hal-01787133 \\ https://hal.science/hal-01787133}

Submitted on 7 May 2018

HAL is a multi-disciplinary open access archive for the deposit and dissemination of scientific research documents, whether they are published or not. The documents may come from teaching and research institutions in France or abroad, or from public or private research centers.
L'archive ouverte pluridisciplinaire HAL, est destinée au dépôt et à la diffusion de documents scientifiques de niveau recherche, publiés ou non, émanant des établissements d'enseignement et de recherche français ou étrangers, des laboratoires publics ou privés. 


\title{
Ion Velocity Distribution Function Investigated Inside an Unstable Magnetized Plasma Exhibiting a Rotating Nonlinear Structure
}

\author{
C. Rebont, N. Claire, ${ }^{*}$ Th. Pierre, and F. Doveil \\ PIIM, UMR6633 CNRS/Université de Provence, case 321, centre universitaire de Saint-Jérôme, 13397 Marseille, France.
} (Received 6 December 2010; published 3 June 2011)

\begin{abstract}
The frequent situation where a strongly nonlinear rotating structure develops in a linear magnetized plasma column is investigated experimentally with emphasis on the ion velocity distribution function (IVDF). Most often, a mode $m=2$ appears exhibiting a large density and potential perturbation with angular frequency slightly above the ion cyclotron frequency. For the first time the spatiotemporal evolution of the IVDF is studied using time-resolved laser induced fluorescence to explore the ion's interaction with the nonlinear wave propagating inside the column and at the origin of plasma transport outside the limiter. The ion fluid exhibits an alternance from azimuthal to radial velocity due to the electric field inside the rotating structure. A fluid model also allows us to locally reconstruct the self-consistent electric field evolution which contradicts all existing theories.
\end{abstract}

PACS numbers: 52.20.-j, 52.25.Xz, 52.35.Mw, 52.35.Ra

As in fluid turbulence [1], the formation, motion, and decay of large scale fluctuation structures is a key issue in plasma turbulence [2]. Inside a plasma, the detailed knowledge of the velocity distribution of the ionized species is very important because it determines the stability of the medium. Moreover, the strong anisotropy induced by the application of a magnetic field gives rise to several effects connected to the cyclotronic motion of the particles. Until recently, only crude methods allowed us to estimate the distribution function of the ions. The time-resolved laser induced fluorescence (LIF) technique applied to plasma ions has recently started a new trend in this field of plasma equilibrium and stability. Indeed, one may compare the benefits of this technique to the particle image velocimetry used in classical neutral fluids. The precise determination of the velocity field in both plasma and fluid media leads to a better knowledge of the dynamics of coherent structures.

In this Letter, we investigate the motion of the magnetized ions under the action of the self-consistent electric field established in a rotating electrostatic plasma structure. The measurements reported here highlight the complexity of the mechanisms leading to the evolution of the ion velocity distribution and give new perspectives in this field as the results do not strengthen existing theories.

Unlike fusion experiments [2], small-size lowtemperature magnetized plasma discharges [3-7] can be operated in a steady state, and the available spatially and temporally resolved diagnostics can provide the wealth of information necessary to make progress in the understanding of turbulence in magnetized plasmas [8]. Density and potential fluctuations measured using arrays of electric probes [9-11] and turbulence imaging with ultrafast cameras $[12,13]$ coupled with data analysis methods [14] and conditional averaging recently played a prominent role.
We report here a direct measurement of the ion dynamics in an unstable magnetized plasma column using timeresolved LIF [15] giving with a high accuracy the ion velocity distribution function (IVDF), temporally and spatially resolved [16]. Electric probe measurements and fast camera imaging have been previously used in the MISTRAL plasma device $[9,17,18]$. It has been shown that the transition from regular to unstable or turbulent regimes can be externally controlled by biasing the end plate of the column. The plasma parameters are adjusted to produce a strongly nonlinear coherent structure, associated to an $m=2$ mode, leading to the rotation of two spiral arms of plasma outside the core plasma. These spiral arms are radially expanding out of the central ionization region. In this regime, time-resolved LIF is applied to the investigation of the temporal and spatial evolution of IVDF inside the central structure.

The MISTRAL device has already been described elsewhere [9]. In a first large source chamber, energetic ionizing electrons are produced and accelerated towards the grounded anode. A magnetic multipolar structure increases the confinement time of the ionizing electrons. The second chamber in which measurements are made is a tube containing a linear plasma column under axial magnetic field. The column is $1 \mathrm{~m}$ in length and $40 \mathrm{~cm}$ in diameter. The uniform magnetic field is produced by a set of 19 coils with a current fixed in order to produce a magnetic field of $16 \mathrm{mT}$. The working gas pressure is typically $2.5 \times 10^{-4}$ mbar in argon.

The linear magnetized plasma column is separated from the source chamber by a biased grid covering a circular $10 \mathrm{~cm}$ aperture in the center of a metallic diaphragm limiter. With grounded anode, the separating grid is most often biased at $4 \mathrm{~V}$, slightly below the plasma potential inside the source chamber. At the end of the column, an 
end-grid collector is positively biased at $20 \mathrm{~V}$. These two biasing values are defining the plasma potential on the axis of the column. On the other hand, the outer cylindrical wall is grounded, inducing a radial electric field pointing inward, and should lead to the $E \times B$ rotation of both ionizing and plasma electrons. In general, the steady-state system exhibits a self-consistent radial sheath whose characteristics are determined by the flux of incoming charges, the collection of positive and negative species on the end plate, and the biasing of the limiters [19]. It is important to note that the biasing of the axial end grid determines the frequency of the unstable mode. As a consequence, this instability cannot be described as a diamagnetic drift wave as recorded in similar devices at higher magnetic field. In fact, this type of nonlinear rotating structure with spiral arms was detected long ago in magnetized plasmas [20]. An analytical study has exhibited the main characteristics [21] of these structures existing when a sheared radial electric field is established. A detailed measurement of sheared drift waves' structures has also been reported using probes [22] and ultrafast imaging [23]. Recently, a detailed study proposed a kinetic description of the unstable low-frequency waves in a magnetized plasma column under rotation induced by the injection of energetic electrons [24].

The plasma parameters are $n_{e}=10^{10} \mathrm{~cm}^{-3}, \nu_{\mathrm{ci}}=$ $6 \mathrm{kHz}, T_{e}=2-3 \mathrm{eV}$. In these conditions a strongly nonlinear coherent $m=2$ mode develops in the plasma at $\nu=7.5 \mathrm{kHz}>\nu_{\text {ci }}$. Two spiral arms expand out in the scrape-off layer behind the limiter. This is best shown in Fig. 1(a) which depicts the plasma density map obtained using the 2D scan of a moving Langmuir probe [17].

Our investigation relies on the LIF diagnostics [15] using a tunable dye laser chopped by an acousto-optic modulator and pumped by a continuous argon laser. The spectral purity of the dye laser is very high $(0.5 \mathrm{MHz})$ and the central frequency is electronically tunable. Three argon atomic levels are used: the $3 d^{2} G_{9 / 2}$ metastable level is excited at $611 \mathrm{~nm}$ to the $4 p^{2} F_{7 / 2}$ level whose decay at $460.9 \mathrm{~nm}$ to the $3 s^{2} G_{9 / 2}$ level is observed. With this method, the IVDF
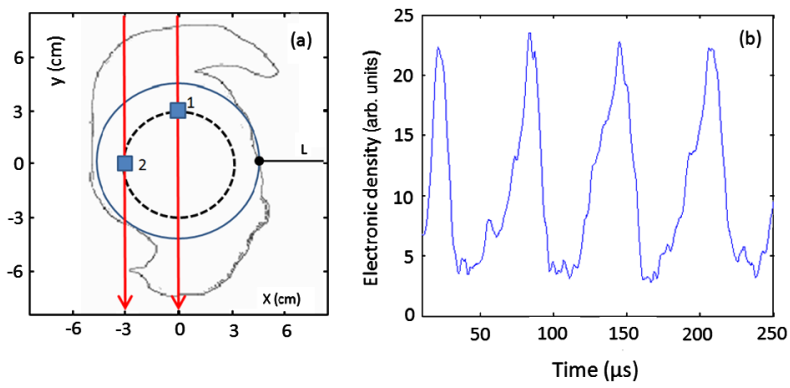

FIG. 1 (color online). (a) Contour map of the electron density in a section of the magnetized plasma column [17]. (b) Plasma density versus time measured by a Langmuir probe. Vertical lines show the two laser beams. $L$ denotes the fixed Langmuir probe. The circle (continuous line) indicates the limiter. The dashed circle indicates the radial location of the measurements: 1 and 2 denote positions of LIF measurements. along the laser beam can be recorded with an excellent spatial resolution [25-27]. The laser beam is fired inside the plasma along two vertical lines shown in Fig. 1(a). The fluorescence radiation emitted by the probed ions is collected in a direction perpendicular to the laser beam by an optical system whose aperture sets the axial and radial resolution at about $1 \mathrm{~cm}$. This value is close to the ion Larmor radius with thermal velocity $v_{\text {thi }}=450 \mathrm{~m} / \mathrm{s}$. A $1 \mathrm{~nm}$ bandpass optical filter selects the fluorescence radiation which is detected by a photomultiplier tube. A multichannel scaler [28] records the counting rate of events. With repetitive measurements, the scan in laser frequency is synchronized with the rotation of the structure inside the plasma and multiple scans are summed up. The temporal resolution is $10 \mu \mathrm{s}$ and the duration of one record is about 20 min. Figure 1(a) shows the two positions located on a circle of radius $r_{F}=3 \mathrm{~cm}$ where measurements are made inside the device at an axial distance $35 \mathrm{~cm}$ from the collector. No LIF measurements are made outside the ionization region where the fast imaging exhibits the spiral structure [13]. Indeed, in this region, the low signal to noise ratio would imply very long signal averaging compared to the thermal drift of the plasma parameters.

Strong density fluctuations are recorded on a probe located at radius $r=5 \mathrm{~cm}$, at the edge of the central plasma column as shown in Fig. 1(b). The angular frequency of the $m=2$ mode structure is about $\omega=$ $45000 \mathrm{rad} / \mathrm{s}$. Time-resolved LIF measurements are shown in Fig. 2 with triggering by the Langmuir probe signal. Because measurements at positions 1 and 2 are made at the

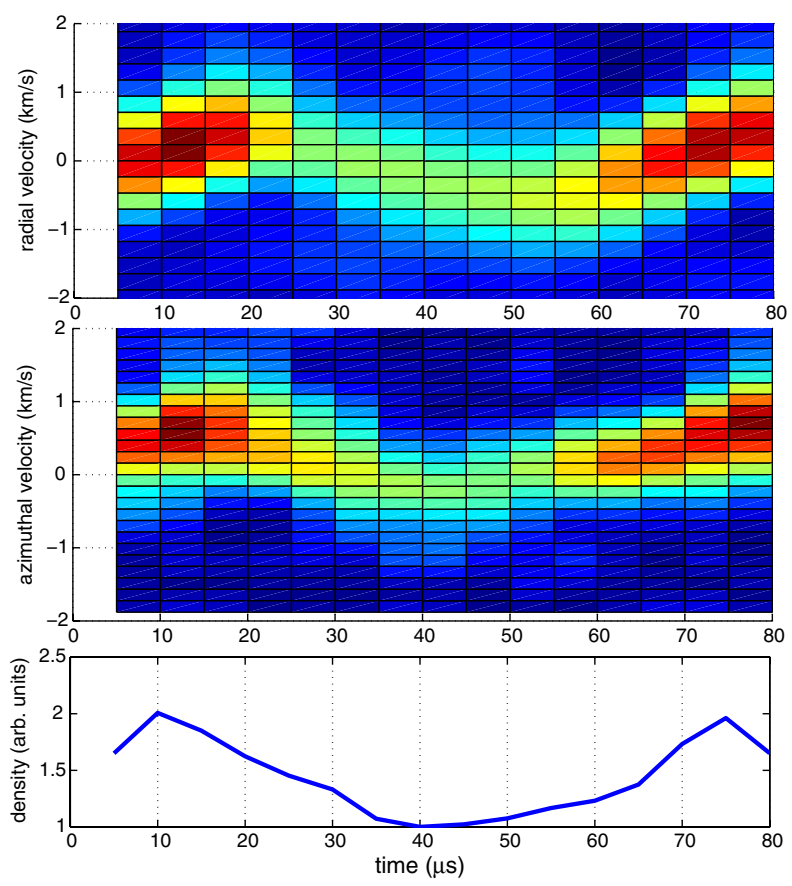

FIG. 2 (color online). Temporal contour plot of the IVDF at $r_{F}=3 \mathrm{~cm}$ measured along radial direction (top) and along azimuthal direction (middle). The temporal density modulation is shown for comparison (bottom). 
same radius, assuming that the ion velocity measurements along the laser beam at point 1 correspond to ion radial velocity and that the coherent $m=2$ mode angular frequency is constant, the measurement of the azimuthal ion velocity at point 2 can be considered as the temporally shifted azimuthal velocity measurement at point 1 . Temporal synchronization between the two measurement locations is always carried out by correlating the ion density perturbations.

Figure 2 shows the radial (top) and azimuthal (middle) temporal contour plot of the IVDF at position 1. The temporal density modulation (obtained by integrating the measured IVDF over velocity) in the probed volume is shown for comparison (bottom). Let us assume that a wave propagating inside the plasma is associated to the rotating structure observed in the limiter region. When the azimuthal analysis of the wave-particle interaction is performed, it is necessary to consider IVDF in the frame moving at the wave phase velocity $v_{\theta}=\omega r_{F}=1.4 \mathrm{~km} / \mathrm{s}$. In the middle plot of Fig. 2, the observed temporal modulation of the azimuthal velocity distribution can be interpreted in the wave moving frame as a strong azimuthal modulation inside the rotating wave with minimum velocity at the peak value of density (deceleration of the ions in the moving frame). It is thus clearly seen that the ions are decelerated when they progressively overcome the positive potential associated with the structure. The measured amplitude of this deceleration of the ions along the azimuthal direction on the leading edge of the potential structure can be used to calculate the local electrostatic potential in the moving frame of the structure. On the other hand, the radial analysis can be made in the laboratory frame because no steady radial movement of the electrostatic structure exists. We also observe on the upper plot of Fig. 2 a modulation of the radial velocity of the ions passing the wave. More precisely, an outward displacement of the ions is recorded in the leading front of the wave potential structure as a consequence of $E \times B$ deflection. The calculation of the IVDF modification under this effect is not that simple because of the low magnetization of the ions.

Moreover, the fact that the plasma is continuously created by ionizing electrons leads to a complex situation. At any time, ions are created at rest and they are accelerated under the local electric field. This leads to a continuous filling of the IVDF in the low energy range of the IVDF. The detailed kinetic effects detected in this electrostatic interaction between the moving potential structure and the charged particles cannot be reported in the frame of this Letter.

Using the same trick as before and since we are dealing with a coherent $m=2$ mode, azimuthal derivative at the measurement point can be replaced by temporal derivative $\frac{\partial}{\partial \theta}=-\frac{1}{\omega} \frac{\partial}{\partial t}$ where $\omega=2 \pi \nu$ is the symmetrical $m=2$ mode pulsation. The spatial evolution of the ion mean velocity can be estimated owing to the rigid body rotation of the structure, with the azimuthal velocity $v_{\theta}$. The temporal evolution is then converted in spatial evolution through multiplication by this velocity. Figure 3 thus shows the reconstruction of the instantaneous ion fluid velocity vector (continuous vectors) in a cross section of the column for the radius $r_{F}$. The velocity is mainly either azimuthal or radial. A laser misalignment could explain the small angle recorded between the measured velocities and the radial or azimuthal direction. As already mentioned before, through wave-particle interaction analysis, a striking result is that a density minimum [at time $t=40 \mu \mathrm{s}$ in Fig. 1(b)] corresponds to a radial ion ejection velocity (around $400 \mathrm{~m} / \mathrm{s}$ ) whereas a density maximum [at time $t=10 \mu(\mathrm{s})$ or $70 \mu(\mathrm{s})]$, when a spiral arm is passing by, mainly corresponds to an azimuthal ion velocity (around $650 \mathrm{~m} / \mathrm{s}$ ) lower than $v_{\theta}$. This was never observed before and cannot be explained by existing theories.

We recall fluid equations for a collisionless plasma in cylindrical coordinates, integrated over axial coordinate, and neglecting the stress tensor $\pi$ :

$$
\begin{gathered}
m_{i} n_{i}\left(\frac{\partial v_{f r}}{\partial t}+v_{f r} \frac{\partial v_{f r}}{\partial r}+\frac{v_{f \theta}}{r} \frac{\partial v_{f r}}{\partial \theta}-\frac{v_{f \theta}^{2}}{r}\right) \\
=q n_{i}\left(E_{r}+v_{f \theta} B_{z}\right)-(\nabla P)_{r}, \\
m_{i} n_{i}\left(\frac{\partial v_{f \theta}}{\partial t}+v_{f r} \frac{\partial v_{f \theta}}{\partial r}+\frac{v_{f \theta}}{r} \frac{\partial v_{f \theta}}{\partial \theta}+\frac{v_{f \theta} v_{f r}}{r}\right) \\
=q n_{i}\left(E_{\theta}-v_{f r} B_{z}\right)-(\nabla P)_{\theta},
\end{gathered}
$$

where the subscripts $r$ and $\theta$ refer to radial and azimuthal coordinates; $m_{i}$ is the ion mass; $\vec{v}_{f}$ the fluid velocity; $n_{i}$ the ion density; $\vec{B}=\vec{B}_{z} \vec{e}_{z}$ the constant axial magnetic field in the linear plasma column; $q=1.6 \times 10^{-19} \mathrm{C}$ the ion charge; $P=n T$ the scalar pressure with $T$ the temperature in energy unit. Using Eqs. (1) and (2) the electric field $\vec{E}$ can be deduced from space and time-resolved ion velocity and density measurements.

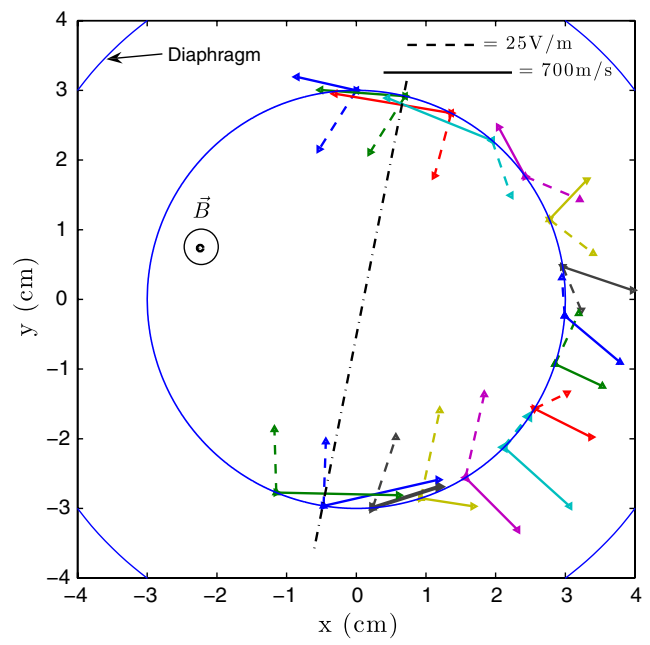

FIG. 3 (color online). Reconstruction of the instantaneous ion mean velocity (continuous vectors) and electric field (dashed vectors). The dot-dashed line indicates the instantaneous density maximum. 
(a)

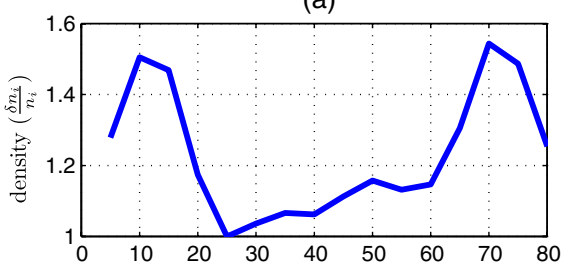

(b)

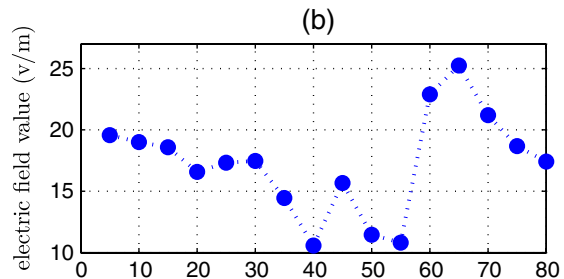

(c)

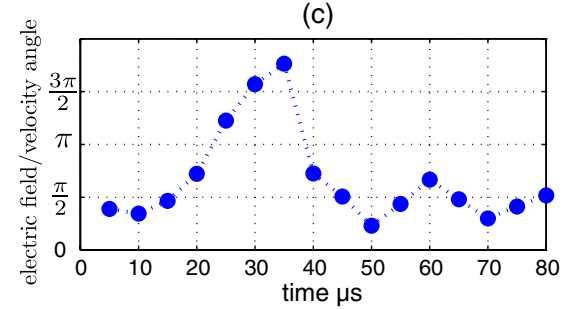

FIG. 4 (color online). Time variation of (a) relative ion density, (b) electric field, (c) angle between electric field and velocity.

Figure 3 also allows us to follow the spatial reconstruction of the local electric field. For its azimuthal component we observe a good qualitative agreement with the previously described wave-particle interaction. The fluid results are analyzed in Fig. 4(a) which shows the time evolution of the ion density measured by LIF, while Fig. 4(b) [Fig. 4(c)] shows the time variation of the electric field amplitude [the angle between the electric field and the ion mean velocity]. On the leading edge of the potential structure, a large variation of the electric field is recorded with a change from 10 to $25 \mathrm{~V} / \mathrm{m}$ in $10 \mu \mathrm{s}$, values that can also be obtained through wave-particle interaction and ion energy conservation in the rotating wave. Whereas the electric field angle with respect to ion velocity is $\frac{\pi}{2}$ on the main part of the period, consistent with $E \times B$ ion fluid drift, on the trailing edge, a variation $\pi$ is seen, related to the fact that ion velocity is strongly time dependent.

Although predominant at the edge as obtained by making the same LIF measurement at a position $r=5 \mathrm{~cm}$, the observed temporal perturbation is not limited to the edge of the plasma column, but it is also associated with a fluctuation of the entire plasma column as was already measured in similar devices $[5,6,10]$. To complete the investigation, we also carried out axial IVDF measurements by firing the laser beam along the plasma column axis. In this case we do not observe any fluctuation of the mean ion fluid velocity, but only the expected ion density fluctuation at the unstable frequency $2 \nu=15 \mathrm{kHz}$ as shown in Fig. 1(b).

In conclusion, using time-resolved LIF diagnostic, we report the first detailed measurement of the ion features inside an $m=2$ nonlinear mode in a linear magnetized plasma. The naive image of a plasma exhibiting a global rotation and commonly found in the literature is invalidated by our results. These are, on the other hand, consistent with the propagation of a strongly nonlinear wave. This wave exhibits a spiral structure probably due to the existence of shear radial electric field outside the ionization zone delimited by the limiter. We observed a predominantly azimuthal ion velocity in the density crest and a radial velocity in the density dip. The angular frequency of the $m=2$ structure is always larger than the mean angular frequency of the ion fluid. The time-resolved measurements have shown that the angular frequency is maximum on the crest of the wave. No ion heating is recorded inside the core plasma column in contrast with previously published results [29]. The reported results clearly show that the implementation of the time-resolved LIF diagnostic opens new interesting prospects for the analysis of ion transport across a hot magnetized plasma. Further measurements will be made inside spiral arms to obtain a complete IVDF map.

This work was partly supported by Agence Nationale de la Recherche (ANR-07-BLAN-0187-01, PHOTONITER).

*nicolas.claire@univ-provence.fr

[1] U. Frisch, Turbulence: The Legacy of A. N. Kolmogorov (Cambridge University Press, Cambridge, England, 1995).

[2] G. R. Tynan et al., Plasma Phys. Controlled Fusion, 51, 113001 (2009).

[3] Th. Pierre et al., Rev. Sci. Instrum. 58, 6 (1987).

[4] R. G. Greaves et al., Plasma Phys. Controlled Fusion 34, 1253 (1992).

[5] E. Gravier et al.,Phys. Plasmas 11, 529 (2004).

[6] S. Klose et al., Contrib. Plasma Phys. 41, 467 (2001).

[7] E. Wallace et al., Rev. Sci. Instrum. 75, 5160 (2004).

[8] T. Yamada et al., Nature Phys. 4, 721 (2008).

[9] Th. Pierre et al., Phys. Rev. Lett. 92, 065004 (2004).

[10] E. Gravier et al., Phys. Plasmas 15, 122103 (2008).

[11] O. Grulke et al., Phys. Plasmas 6, 788 (1999).

[12] S. J. Zweben et al., Phys. Plasmas 9, 1981 (2002).

[13] C. Brault et al., Phys. Lett. A 360, 299 (2006).

[14] S. Magni et al., Phys. Rev. E 72, 026403 (2005).

[15] D. N. Hill et al., Rev. Sci. Instrum. 54, 309 (1983).

[16] N. Claire et al., Rev. Sci. Instrum. 72, 4372 (2001).

[17] M. Matsukuma et al., Phys. Lett. A 314, 163 (2003).

[18] S. Jaeger et al., Phys. Plasmas 16, 022304 (2009).

[19] R. D. Hazeltine, Phys. Fluids B 1, 2031 (1989).

[20] E. I. Dobrokhotov et al., Nucl. Fusion 9, 143 (1969).

[21] M. Kono and M. Y. Tanaka, Phys. Rev. Lett. 84, 4369 (2000).

[22] C. Schröder et al., Phys. Plasmas 11, 4249 (2004).

[23] G. Y. Antar et al., Phys. Plasmas 14, 022301 (2007).

[24] Yu. N. Yeliseyev, Plasma Phys. Rep. 32, 927 (2006).

[25] N. Claire et al., Phys. Plasmas 13, 062103 (2006).

[26] I. Uzun-Kayamk et al., Phys. Plasmas 13, 112108 (2006).

[27] A. Diallo et al., Phys. Plasmas 13, 055705 (2006).

[28] B. Pélissier et al., Rev. Sci. Instrum. 67, 3405 (1996).

[29] F. Anderegg et al., Phys. Rev. Lett. 57, 329 (1986). 